
\title{
$\begin{array}{ll}\text { Research Square } & \begin{array}{l}\text { Preprints are preliminary reports that have not undergone peer review. } \\ \text { They should not be considered conclusive, used to inform clinical practice, } \\ \text { or referenced by the media as validated information. }\end{array}\end{array}$
}

\section{An Optimal Control Problem of Chemotherapy In Presence of Gene and Immunotherapy}

kaushik dehingia ( $\square$ kaushikdehingia17@gmail.com )

Gauhati University

\section{Research Article}

Keywords: Cancer eradication model, Gene Therapy, Chemotherapy, Immunotherapy, Stability, Optimal Control

Posted Date: June 9th, 2021

DOI: https://doi.org/10.21203/rs.3.rs-605711/v1

License: (c) (i) This work is licensed under a Creative Commons Attribution 4.0 International License. Read Full License

Version of Record: A version of this preprint was published at AIMS Mathematics on August 9th, 2021. See the published version at https://doi.org/\%2010.3934/math.2021669. 


\title{
An Optimal Control Problem of Chemotherapy In Presence of Gene and Immunotherapy
}

\author{
Kaushik Dehingia ${ }^{1}$, Hemanta Kumar Sarmah ${ }^{1}$, Kamyar Hosseini $^{2 *}$, Soheil \\ Salahshour ${ }^{3}$ \\ ${ }^{1}$ Department of Mathematics, Gauhati University, Assam, India \\ ${ }^{2}$ Department of Mathematics, Rasht Branch, Islamic Azad University, Rasht, Iran \\ ${ }^{3}$ Faculty of Engineering and Natural Sciences, Bahcesehir University, Istanbul, Turkey \\ E-mail addresses: ${ }^{1}$ kaushikdehingia17@gmail.com, ${ }^{1}$ nsarmah@hotmail.com, \\ ${ }^{2}$ kamyar_hosseini@yahoo.com, ${ }^{3}$ soheil.salahshour@eng.bau.edu.tr \\ *Author of Correspondence: kamyar_hosseini@yahoo.com
}

\begin{abstract}
In this study, a cancer eradication model involving effector cells in the presence of gene therapy, immunotherapy, and chemotherapy has been proposed. Boundedness and positiveness of the solutions in the respective feasible domains of the proposed model are verified. Conditions for which the equilibrium points of the system are stable have been derived. Amounts of immunotherapy to be injected to a patient for eradication of cancerous tumor cells have been found. To minimize the chemotherapy drug-induced toxicity to the patient, an optimal control problem for the system has been constructed and solved. Numerical and graphical results have been presented. Through the investigation, it was seen that tumor cells can be eliminated in a specific time interval with the control of chemotherapeutic drug concentration.
\end{abstract}

Keywords: Cancer eradication model; Gene Therapy; Chemotherapy; Immunotherapy; Stability; Optimal Control.

2020 AMS Subject Classification: 37M05, 37M10, 37N25, 92B05.

\section{Introduction}

Cancer, the disease which triggers unregulated development of irregular cells in the human body, is one of the main factors of mortality among mankind. As the disorder caused of the disease goes to the tissue level, the task of precise detection at an early stage of the disease becomes a really difficult task. Another obstacle faced at the treatment level is to decide the therapeutic effectiveness of the drugs to be administered. Many researchers all over the world are carrying out their research work for finding out the cause of origin of the disease and for imparting a patient-friendly effective, and successful treatment method.

Mathematical modeling since a long time back has been used as a tool for finding out some solutions to this problem faced of human population [1, 2]. Kuznetsov et al. [3] proposed a model showing the reaction of the cytotoxic $\mathrm{T}$ lymphocyte to the development of the immunogenic tumor. Kirschner and Panetta [4] proposed a model to explore the effects of IL-2 therapy and ACI therapy on tumor-immune dynamics. Kolev et al. [5] presented a model that identified the early phases of a solid tumor or all phases of leukaemia. A variety of clinical trials have been performed to investigate the impact of the immune system on the destruction of tumor cells in the body by inducing the host's own immune response to destroy cancer cells $[6,7]$. As the tumor grows in the human 
body, the immune system seeks to recognize and destroy the tumor cells as soon as possible. In [8], Rihan et al. examined the interactions among tumor cells, immune-induced cells, and interleukin-2 (IL-2) cytokines. Moreover, several works have been carried out by different researchers by inducing delay effects on various factors in the process of cancer evolution and propagation. In [9], the author considered a tumor-immune interaction model inducing three time delays to describe the proliferation of tumor cells, the process of effector cell growth stimulated by tumor cells, and the differentiation of immune effector cells. Khajanchi and Banerjee modified the Kuznetsov model [3] by implementing a discrete time lag in tumor-immune interactions. They have also been carried out the bifurcation analysis and showed that Hopf bifurcation occurs when the time lag passes a critical value [10]. Khajanchi et al. [11] carried out a modified delayed model which exhibits periodic solutions and rich chaotic dynamics. Furthermore, in the work [12], S. Khajanchi analyzed the bifurcation of the above model [11] and determined the direction and stability of Hopf bifurcating periodic solutions. Ghosh et al. modified Khajanchi et al. model [11] by considering two time delays, one for interactions between tumor cells and immune cells and another for the action of immune cells on tumor cells. They have calculated the range of delays for which immune cells hinder the tumor cells [13]. A review work of some recent delayed work on tumor-immune interactions was described by S. Ruan [14]. Moreover, a discussion on a three-delayed tumor-immune system interaction model have been carried out.

Chemotherapy is an effective method to fight against cancer [15]. Yet, it has a substantial negative effect as induction of chemotherapeutic drugs depletes the immune system and makes the patients vulnerable to harmful infections. Thus, after the immunedepleting phase of chemotherapy, it becomes an absolute necessity to improve the immune system of the patient. Therefore, it is a difficult challenge during the chemotherapy process to preserve a stable immune system and combat cancer successfully [16]. Therefore, in the present day, keeping this point in view, researchers are interested in a combination of two or more therapies in the chemotherapeutic treatment protocols. Until now, a number of significant works have been carried out in the field of cancer chemotherapeutic modeling. de Pillis et al.[17] updated their previously proposed model [16] with the inclusion of NK cells, $C D 8^{+} T$ cells, and IL-2 therapy. Their results revealed that while eliminating cancerous tissue in the presence of chemotherapy, immunotherapy is helpful for low immune efficacy patients. Effect of anti-angiogenic agents in controlling cancer with chemotherapy has been demonstrated in [18]. An appropriate optimal combination treatment of vaccine therapy and chemotherapy for controlling cancer dynamics has been presented in [19]. In consideration of radiotherapy as a treatment of cancer followed by chemotherapy, a periodic mathematical has been proposed by Liu and Yang [20]. Guiraldello et al. presented a mathematical model involving partial differential equations to show the behavior of tumor development in the presence of two chemotherapy protocols, Maximum Tolerated Dose (MTD) and metronomic as well as two methods of drug delivery. They have also evaluated the effectiveness of both protocols [21]. Pang et al. explored the effect of a combination of immunotherapy and chemotherapy, single chemo, and immunotherapy in their work [22]. Rodrigues et al. [23] explored a model by considering chemo-immunotherapy as a treatment to reduce unwanted cytotoxic effects on immune cells of a cancer patient. In [24], the authors presented a model for the growth of the immune system during chemotherapy. Alqudah [25] introduced a new mathematical model for the treatment of cancer as a combination of chemotherapy and stem cell 
therapy.

Cancer chemotherapy is usually administered on a cyclic basis. When an anti-cancer medicine is injected into the body, all harmless cells also get destroyed along with malignant cells. As a result, white blood cell counts decline to low rates and life-threatening fever may occur [15]. Thus, control of the chemotherapy dosage becomes a very critical aspect in cancer chemotherapy design $[26,27]$.

At the molecular level, cancer can be characterized as a genetic defect in cell cycle. Gene therapy is a common form of immunotherapy that strengthens the body's immunity and aims to cure genetic conditions. By inserting a well-designed gene into the cells of the patients, correction can be carried out in the inborn inaccuracy of metabolism, inherited hereditary abnormality, etc.[28].

Ongoing research of clinical studies are now developing in cancer eradication policies by incorporating two or more drugs [29]. Application of chemotherapy and gene therapy is now in operation which has demonstrated that this type of treatment reduces the primary tumor, avoids intra-peritoneal metastases and restricts harmful side effects [30]. Application of TRAIL gene therapy and chemotherapy is also successful in the diagnosis of metastatic diseases [31]. Anti-cancer gene therapy under review is generally conducted in a two-phase cycle. The first step is a successful search for chemo-resistant cells during chemotherapy, which results in an increased proportion of transfected cells. Population of the transfected cells must surpass that of the mutant cells in order to maintain the optimum relationship between the species necessary for the bystander effect. The second step consists of a negative selection step accomplished by ganciclovir injection. Such cells that are vulnerable to antiviral drug treatment through transfection or bystander effect will undergo activated apoptosis [32]. In 2013, Tsygvinsev et al. [33] first proposed a mathematical model of gene therapy for cancer treatment by modifying an already investigated model [4]. We have considered a modified version of the model proposed by Tsygvintsev et al. [33] to study in this paper.

Controlling of drugs in the treatment of cancer patients is a big problem for researchers. Many researchers are working on optimal drug control for cancer treatment. Khajanchi and Ghosh designed a model based on Kuznetsov et al. model [3] with two types of external treatments, one is ACI therapy for anti-tumor activity and another is IL-2 therapy for boosting the immune system to fight against tumor [26]. Moreover, they have found the optimal combination treatment for tumor reduction. Another optimal chemotherapeutic strategy for a tumor growth model has been carried out by Sharma and Samanta [27]. Rihan et al. established from their model [34] that the performance of the combination therapy protocol of immuno-chemotherapy is better than the standard protocol of chemotherapy alone. d'Onofrio et al. [35] formulated a model for the optimal scheduling of angiogenic inhibitors in combination with a chemotherapeutic agent. In the work [36], the author explored the dynamical behaviour of the glioma-immune interaction in conjunction with an optimally controlled immunotherapeutic drug ACI. By considering an abstract mathematical model of cancer chemotherapy, Leszczynski et al. investigated an optimal control problem for a single drug and when various pharmacodynamic (PD) and pharmacokinetic (PK) models are included in the modeling [37]. Recently, the authors of [38] designed an optimal model for chemotherapy treatment to 
find the best rate of drug injection during treatment. In order to find better outcomes in drug controlling for therapeutic cancer model, Najafi et al. [39] introduced an optimal control homotopy perturbation method. Rihan et al. [40] studied an extended work of [4] to a delayed model of tumour-immune interactions and then find the optimal protocol of immuno-chemotherapy which reduces the tumor load in a few months of therapy. In the work [41], the author performed a detailed analysis to find the optimal regimens of combined chemo-immunotherapy for a PKPD cancer evolution model. Das et al. [42] analyzed an optimal control problem of immuno-chemotherapeutic drugs for a delayed tumor-immune model. In this article, we proposed a modified cancer gene therapy model in the presence of immunotherapy, and chemotherapy. To control the chemo-drug toxicity effect on the patient, we also construct an optimal control problem for the proposed model.

In Section 2, we have given a description of the model about which we have investigated in the subsequent sections.

\section{Model Description}

We already mentioned several research works on the applications of chemotherapy and gene therapy in the treatment of cancer. In work [33], the authors showed that high levels of immunotherapy and 'cancer clearance term' of gene therapy would be able to clear cancer optimally from patients' body. Lestari et al. [43] studied the local stability analysis of the proposed model [33]. In [44], the authors estimated the external parameters of the model [33] for which the growth of tumor cells remains under control. For further study on the applications of gene therapy in cancer treatment models, the authors would like to refer to the works $[33,43]$ to the reader.

High dose of immunotherapy to a patient can induce negative response such as fever, chills, weakness, nausea etc. [45, 46]. Therefore, in order to reduce these side effects, in this work, we reconstruct the earlier proposed model [33] in combination of chemotherapy and gene therapy. The model is built on the basis of interactions among tumor cells, effector immune cells, gene therapy, immunotherapy and the chemotherapeutic drugs. The assumptions imposed on the model are:

- Gene therapy detects how tumor cells are different from normal cells and this can be described by the term $q M$ with a fixed antigenicity $q$.

- Effector immune cells can proliferate by itself with proliferation rate $p$. Effector immune cells also has natural death rate $\mu$.

- External sources of immunotherapy $s(t)$ (TIL-therapy) boost the immune system, which is time dependent.

- Cancer cell can grow logistically in absence of immmune cells and treatments.

- The interaction of effector cells and cancer cells during gene therapy is considered according as Michaelis-Menten form $-\frac{a I M}{g+M}$ which is usually used for describing anti-tumor immune responses [4]. a represents the cancer clearance rate during gene therapy. 
- Chemotherapy can kill both the cell populations viz. cancer and effector immune cells with different constant rates.

The flowchart of the model is depicted in Fig 1.

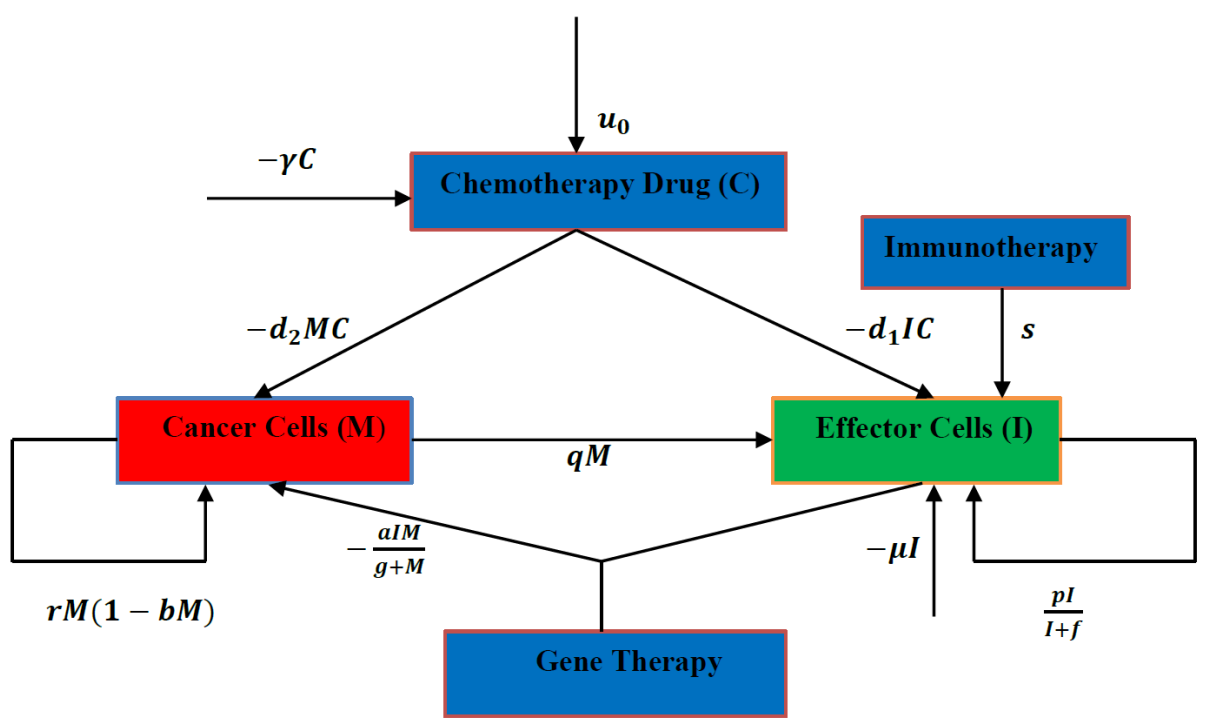

Figure 1: Flowchart for the system (2.1)

Considering above mentioned assumptions and the flowchart, the model [33] can be reformulated as:

$$
\begin{aligned}
\frac{d I}{d t} & =q(t) M+\frac{p I}{I+f}-\mu I+s(t)-d_{1} I C \\
\frac{d M}{d t} & =r(t) M(1-b M)-\frac{a(t) I M}{g+M}-d_{2} M C \\
\frac{d C}{d t} & =u_{0}-\gamma C
\end{aligned}
$$

The first equation represents the dynamics of the effector immune cells $I(t) . q(t) M$ represents the cancer antigenicity during gene therapy which gives strong immunity in the presence of cancer. As the effector cells proliferate itself, so it can be represented by the term $\frac{p I}{I+f}$. $-\mu$ represents the half life of effector cells. The term $s(t)$ is used for describing immunotherapy which boost the immune system. $-d_{1} I C$ is the degradation of effector cells which get killed during chemotherapy.

The second equation describes the variability of the cancerous tumor cells $M(t)$. The term $r(t) M(1-b M)$ represents the logistic growth of cancer cells. $-\frac{a(t) I M}{g+M}$ describes the interaction between effector cell and cancer cell during gene therapy. The term $-d_{2} M C$ indicates the degradation of cancerous cells by chemotherapy.

The last equation is the continuous drug administration of chemotherapy, where $u_{0}$ represents the initial dose of chemotherapeutic drug given and $\gamma$ is the per capita decay 
rate of the chemotherapeutic drug.

In the following section, we have discussed about the positiveness and boundedness of the solutions of the system (2.1).

\section{Basic Characteristics of the Solutions}

Theorem 3.1. Associated with initial conditions $I(0) \geq 0, M(0) \geq 0$ and $C(0) \geq 0$, all the solutions of the system (2.1) exist in $\mathbb{R}_{+}^{3}$ and $I(t) \geq 0, M(t) \geq 0$ and $C(t) \geq 0$ for all $t \geq 0$.

Proof. It is clear that the uniformly continuity and locally Lipschitzian conditions holds for R.H.S of the system (2.1) on its domain. Hence, there exists an unique solution $(I(t), M(t), C(t))$ of $(2.1)$ with initial conditions $I(0) \geq 0, M(0) \geq 0, C(0) \geq 0$ on $\mathbb{R}_{+}^{3}$.

Now,

$$
\begin{aligned}
\frac{d I}{d t} & =q M+\frac{p I}{I+f}-\mu I+s-d_{1} I C \geq \frac{p I}{I+f}-\mu I-d_{1} I C \\
\frac{d M}{d t} & =r M(1-b M)-\frac{a I M}{g+M}-d_{2} M C \\
\frac{d C}{d t} & =u_{0}-\gamma C \geq-\gamma C
\end{aligned}
$$

Solving (3.1), we get

$$
\begin{aligned}
I(t) & \geq I(0) e^{\int_{0}^{t}\left\{\frac{p}{I(t)+f}-\mu-d_{1} C(t)\right\} d t} \geq 0 \\
M(t) & =M(0) e^{\int_{0}^{t}\left\{r(1-b M(t))-\frac{a I(t)}{g+M(t)}-d_{2} C(t)\right\} d t} \geq 0 \\
C(t) & \geq e^{-\gamma C} \geq 0
\end{aligned}
$$

Thus, $I(t), M(t), C(t)$ will be positive in $\mathbb{R}_{+}^{3}$ for all $t \geq 0$.

Theorem 3.2. There exists bounded solutions for the model (2.1) subject to initial conditions $I(0) \geq 0, M(0) \geq 0$ and $C(0) \geq 0$

Proof. From the second equation of system (2.1) and using the standard Kamke comparison theory [27] to it, we have

$$
\begin{aligned}
\frac{d M}{d t} & \leq r M(1-b M) \\
\lim _{t \rightarrow \infty} \sup M(t) & \leq \frac{1}{b}
\end{aligned}
$$

The third equation implies that

$$
\begin{aligned}
C(t) & =\frac{u_{0}}{\gamma}+\left[C(0)-\frac{u_{0}}{\gamma}\right] e^{-\gamma t} \\
\Longrightarrow \lim _{t \rightarrow \infty} \sup C(t) & \leq \frac{u_{0}}{\gamma}
\end{aligned}
$$


Now, using the bounds of $\mathrm{M}(\mathrm{t})$ ( $\operatorname{suppose} \sup M(t)=M_{s}$ ), the first equation implies that

$$
\begin{aligned}
\frac{d I}{d t} & \leq q M_{s}+\frac{p I}{I+f}-\mu I+s \\
\frac{d I}{d t} & \leq \kappa+\frac{p I}{I+f} \\
I(t) & \leq I(0)+\int_{0}^{t}\left(\kappa+\frac{p I(s)}{I(s)+f}\right) d s \\
I(t) & \leq I(0)+\kappa t+\int_{0}^{t}\left(\frac{p I(s)}{I(s)+f}\right) d s
\end{aligned}
$$

where $q M_{s}+s=\kappa$ (by assumption this is constant).

By using the generalized Gronwall Lemma [34], we get

$$
I(t)<A,
$$

where $A$ is uniformly bounded. Which completes the proof.

After establishing the positiveness and boundedness of the solutions of system (2.1), we have derived the equilibrium points of the system and discuss the stability conditions in the next section.

\section{Stability Analysis}

We have found the following equilibrium points:

- Healthy equilibrium: $E_{1}\left(I_{1}, 0, \frac{u_{0}}{\gamma}\right), E_{2}\left(I_{2}, 0, \frac{u_{0}}{\gamma}\right)$, where

$$
\begin{array}{|l|l|}
I_{1}=\frac{A_{2}+\sqrt{B_{2}}}{2 \gamma u_{0}} & A_{2}=p \gamma-\mu f \gamma-d_{1} u_{0} \\
I_{2}=\frac{A_{2}-\sqrt{B_{2}}}{2 \gamma u_{0}} & B_{2}=\left(p \gamma-\mu f \gamma-d_{1} u_{0}\right)^{2}+4 s u_{0} \gamma^{2} \\
\hline
\end{array}
$$

- Disease persistent equilibrium: $E_{3}\left(I_{3}, M_{1}, \frac{u_{0}}{\gamma}\right), E_{4}\left(I_{4}, M_{1}, \frac{u_{0}}{\gamma}\right)$, where

$$
\begin{array}{|l|l|}
\hline I_{3}=\frac{A_{3}+\sqrt{B_{3}}}{4\left(r \gamma^{2} b \mu+d_{1} u_{0} r \gamma b\right)} & A_{1}=r \gamma-b g r \gamma-d_{2} u_{0} \\
I_{4}=\frac{A_{3}-\sqrt{B_{3}}}{4\left(r \gamma^{2} b \mu+d_{1} u_{0} r \gamma b\right)} & B_{1}=\left(r \gamma-b g r \gamma-d_{2} u_{0}\right)^{2}-4(r \gamma b)\left(a I \gamma-r \gamma g+d_{2} u_{0}\right) \\
M_{1}=\frac{A_{1}+\sqrt{B_{1}}}{2 r \gamma b} & A_{3}=q \gamma\left(A_{1}+\sqrt{B_{1}}\right)-2 r \gamma^{2} b \mu f+2 p r \gamma^{2} b+2 r s \gamma^{2} b-2 r \gamma b d_{1} u_{0} f \\
& B_{3}=A_{3}^{2}+4\left(2 r \gamma^{2} b \mu+2 d_{1} u_{0} r \gamma b\right)\left\{q \gamma f\left(A_{1}+\sqrt{B_{1}}\right)+2 s f \gamma^{2} r b\right\} \\
\hline
\end{array}
$$

- Disease persistent equilibrium: $E_{5}\left(I_{5}, M_{2}, \frac{u_{0}}{\gamma}\right), E_{6}\left(I_{6}, M_{2}, \frac{u_{0}}{\gamma}\right)$, where

$$
\begin{array}{|l|l}
I_{5}=\frac{A_{4}+\sqrt{B_{4}}}{4\left(r \gamma^{2} b \mu+d_{1} u_{0} r \gamma b\right)} & A_{1}=r \gamma-b g r \gamma-d_{2} u_{0} \\
I_{6}=\frac{A_{4}-\sqrt{B_{4}}}{4\left(r \gamma^{2} b \mu+d_{1} u_{0} r \gamma b\right)} & B_{1}=\left(r \gamma-b g r \gamma-d_{2} u_{0}\right)^{2}-4(r \gamma b)\left(a I \gamma-r \gamma g+d_{2} u_{0}\right) \\
M_{2}=\frac{A_{1}-\sqrt{B_{1}}}{2 r \gamma b} & A_{4}=q \gamma\left(A_{1}-\sqrt{B_{1}}\right)-2 r \gamma^{2} b \mu f+2 p r \gamma^{2} b+2 r s \gamma^{2} b-2 r \gamma b d_{1} u_{0} f \\
& B_{4}=A_{3}^{2}+4\left(2 r \gamma^{2} b \mu+2 d_{1} u_{0} r \gamma b\right)\left\{q \gamma f\left(A_{1}-\sqrt{B_{1}}\right)+2 s f \gamma^{2} r b\right\}
\end{array}
$$


Theorem 4.1. The healthy equilibrium $E_{1}$ exists and locally asymptotically stable if

$$
u_{0}>\frac{\gamma}{d_{1}}\left[\frac{p f}{\left(I_{1}+f\right)^{2}}-\mu\right] \text { and } u_{0}>\frac{\gamma}{d_{2}}\left(r-\frac{a I_{1}}{g}\right)
$$

and another healthy equilibrium $E_{2}$ exists and locally asymptotically stable if

$$
u_{0}>\frac{\gamma}{d_{1}}\left[\frac{p f}{\left(I_{2}+f\right)^{2}}-\mu\right] \text { and } u_{0}>\frac{\gamma}{d_{2}}\left(r-\frac{a I_{2}}{g}\right)
$$

Proof. The variational matrix of the system (2.1) is:

$$
\mathbf{V}=\left(\begin{array}{ccc}
-\mu+\frac{p f}{(I+f)^{2}}-d_{1} C & q & -d_{1} I \\
\frac{-a M}{M+g} & r-2 r b M-\frac{a g I}{(M+g)^{2}}-d_{2} C & -d_{2} M \\
0 & 0 & -\gamma
\end{array}\right)
$$

So, the variational matrix at $E_{1}\left(I_{1}, 0, \frac{u_{0}}{\gamma}\right)$ is

$$
\mathbf{V}\left(\mathbf{E}_{\mathbf{1}}\right)=\left(\begin{array}{ccc}
-\mu+\frac{p f}{\left(I_{1}+f\right)^{2}}-d_{1} \frac{u}{\gamma} & q & -d_{1} I_{1} \\
0 & r-a I_{1}-d_{2} \frac{u}{\gamma} & 0 \\
0 & 0 & -\gamma
\end{array}\right)
$$

Therefore, the eigenvalues of the characteristic equation of $\mathbf{V}\left(\mathbf{E}_{\mathbf{1}}\right)$ are

$$
\lambda_{1}=-\mu+\frac{p f}{\left(I_{1}+f\right)^{2}}-d_{1} \frac{u_{0}}{\gamma}, \lambda_{2}=r-a I_{1}-d_{2} \frac{u_{0}}{\gamma}, \lambda_{3}=-\gamma \text {. Clearly, } \lambda_{3}<0 .
$$

Now, $E_{1}$ is stable if $\lambda_{1}<0, \lambda_{2}<0$; this implies $-\mu+\frac{p f}{\left(I_{1}+f\right)^{2}}-d_{1} \frac{u_{0}}{\gamma}<0, r-a I_{1}-d_{2} \frac{u_{0}}{\gamma}<0$; which gives $u_{0}>\frac{\gamma}{d_{1}}\left[\frac{p f}{\left(I_{1}+f\right)^{2}}-\mu\right]$ and $r<a I_{1}+d_{2} \frac{u_{0}}{\gamma}$.

In the same way, the variational matrix at $E_{2}\left(I_{2}, 0, \frac{u_{0}}{\gamma}\right)$ is

$$
\mathbf{V}\left(\mathbf{E}_{\mathbf{2}}\right)=\left(\begin{array}{ccc}
-\mu+\frac{p f}{\left(I_{2}+f\right)^{2}}-d_{1} \frac{u_{0}}{\gamma} & q & -d_{1} I_{2} \\
0 & r-a I_{2}-d_{2} \frac{u_{0}}{\gamma} & 0 \\
0 & 0 & -\gamma
\end{array}\right)
$$

The eigenvalues of the characteristic equation of $\mathbf{V}\left(\mathbf{E}_{\mathbf{2}}\right)$ are

$$
\lambda_{1}=-\mu+\frac{p f}{\left(I_{2}+f\right)^{2}}-d_{1} \frac{u_{0}}{\gamma}, \lambda_{2}=r-a I_{2}-d_{2} \frac{u_{0}}{\gamma}, \lambda_{3}=-\gamma \text {. Clearly, } \lambda_{3}<0 .
$$

Now, $E_{2}$ is stable if $\lambda_{1}<0, \lambda_{2}<0$; this implies $-\mu+\frac{p f}{\left(I_{2}+f\right)^{2}}-d_{1} \frac{u_{0}}{\gamma}<0, r-a I_{2}-d_{2} \frac{u_{0}}{\gamma}<0$; which gives $u_{0}>\frac{\gamma}{d_{1}}\left[\frac{p f}{\left(I_{2}+f\right)^{2}}-\mu\right]$ and $r<a I_{2}+d_{2} \frac{u_{0}}{\gamma}$.

Theorem 4.2. The disease persistent equilibrium $E_{3}$ exists and locally asymptotically stable if

$$
q>\frac{\left[-\mu+\frac{p f}{\left(I_{3}+f\right)^{2}}-d_{1} \frac{u_{0}}{\gamma}\right]\left[r-2 r b M_{1}-\frac{a g I_{3}}{\left(M_{1}+g\right)^{2}}-d_{2} \frac{u_{0}}{\gamma}\right]}{\left(-\frac{a M_{1}}{g+M_{1}}\right)}
$$

and another disease persistent equilibrium $E_{4}$ exists and locally asymptotically stable if

$$
q>\frac{\left[-\mu+\frac{p f}{\left(I_{4}+f\right)^{2}}-d_{1} \frac{u_{0}}{\gamma}\right]\left[r-2 r b M_{1}-\frac{a g I_{4}}{\left(M_{1}+g\right)^{2}}-d_{2} \frac{u_{0}}{\gamma}\right]}{\left(-\frac{a M_{1}}{g+M_{1}}\right)}
$$

Proof. The variational matrix at $E_{3}\left(I_{3}, M_{1}, \frac{u_{0}}{\gamma}\right)$ is

$$
\mathbf{V}\left(\mathbf{E}_{3}\right)=\left(\begin{array}{ccc}
-\mu+\frac{p f}{\left(I_{3}+f\right)^{2}}-d_{1} \frac{u_{0}}{\gamma} & q & -d_{1} I_{3} \\
-\frac{a M_{1}}{g+M_{1}} & r-2 r b M_{1}-\frac{a g I_{3}}{\left(M_{1}+g\right)^{2}}-d_{2} \frac{u_{0}}{\gamma} & -d_{2} M_{1} \\
0 & 0 & -\gamma
\end{array}\right)
$$


The characteristic equation of $\mathbf{V}\left(\mathbf{E}_{\mathbf{3}}\right)$ is

$$
(-\gamma-\lambda)\{(C-\lambda)(A-\lambda)-B q\}=0,
$$

where, $A=-\mu+\frac{p f}{\left(I_{3}+f\right)^{2}}-d_{1} \frac{u_{0}}{\gamma}, B=-\frac{a M_{1}}{g+M_{1}}, C=r-2 r b M_{1}-\frac{a g I_{3}}{\left(M_{1}+g\right)^{2}}-d_{2} \frac{u_{0}}{\gamma}$.

From equation (4.2) we get,

$$
\lambda_{1}<\frac{(A+C)+\sqrt{(A+C)^{2}+4(B q-A C)}}{2}, \lambda_{2}<\frac{(A+C)-\sqrt{(A+C)^{2}+4(B q-A C)}}{2}, \lambda_{3}<0 \text {. Clearly, }
$$

$\lambda_{3}<0 . E_{3}$ is stable if $\lambda_{1}<0, \lambda_{2}<0$, which gives, $B q-A C<0 \Longrightarrow q>\frac{A C}{B}$. This follows the result.

The variational matrix at $E_{4}\left(I_{4}, M_{1}, \frac{u_{0}}{\gamma}\right)$ is

$$
\mathbf{V}\left(\mathbf{E}_{\mathbf{4}}\right)=\left(\begin{array}{ccc}
-\mu+\frac{p f}{\left(I_{4}+f\right)^{2}}-d_{1} \frac{u_{0}}{\gamma} & q & -d_{1} I_{4} \\
-\frac{a M_{1}}{g+M_{1}} & r-2 r b M_{1}-\frac{a g I_{4}}{\left(M_{1}+g\right)^{2}}-d_{2} \frac{u_{0}}{\gamma} & -d_{2} M_{1} \\
0 & 0 & -\gamma
\end{array}\right)
$$

The characteristic equation of $\mathbf{V}\left(\mathbf{E}_{4}\right)$ is

$$
(-\gamma-\lambda)\{(C-\lambda)(A-\lambda)-B q\}=0,
$$

where, $A=-\mu+\frac{p f}{\left(I_{4}+f\right)^{2}}-d_{1} \frac{u_{0}}{\gamma}, B=-\frac{a M_{1}}{g+M_{1}}, C=r-2 r b M_{1}-\frac{a g I_{4}}{\left(M_{1}+g\right)^{2}}-d_{2} \frac{u_{0}}{\gamma}$.

From equation (4.3) we get,

$$
\lambda_{1}<\frac{(A+C)+\sqrt{(A+C)^{2}+4(B q-A C)}}{2}, \lambda_{2}<\frac{(A+C)-\sqrt{(A+C)^{2}+4(B q-A C)}}{2}, \lambda_{3}<0 \text {. Clearly, }
$$

$\lambda_{3}<0 . E_{4}$ is stable if $\lambda_{1}<0, \lambda_{2}<0$, which gives, $B q-A C<0 \Longrightarrow q>\frac{A C}{B}$. This follows the result.

Theorem 4.3. The disease persistant equilibrium $E_{5}$ exists and locally asymptotically stable if

$$
q>\frac{\left[-\mu+\frac{p f}{\left(I_{5}+f\right)^{2}}-d_{1} \frac{u_{0}}{\gamma}\right]\left[r-2 r b M_{2}-\frac{a g I_{5}}{\left(M_{2}+g\right)^{2}}-d_{2} \frac{u_{0}}{\gamma}\right]}{\left(-\frac{a M_{2}}{g+M_{2}}\right)}
$$

and another disease persistent equilibrium $E_{6}$ exists and locally asymptotically stable if

$$
q>\frac{\left[-\mu+\frac{p f}{\left(I_{6}+f\right)^{2}}-d_{1} \frac{u_{0}}{\gamma}\right]\left[r-2 r b M_{2}-\frac{a g I_{6}}{\left(M_{2}+g\right)^{2}}-d_{2} \frac{u_{0}}{\gamma}\right]}{\left(-\frac{a M_{2}}{g+M_{2}}\right)}
$$

Proof. Same as above.

The following section is related to the study of the control problem related to our proposed model (2.1), when we administer chemotherapy treatment over a fixed period. For the biomedical perspective, we use the concept of optimal control for the model. This addresses how the combination of chemo-gene-immunotherapy might be an efficient treatment to assist the patient fighting against cancer.

\section{The Control Problem}

To construct and analyze the optimal control problem for our proposed model, we follow the previous works $[26,27,42]$. As a higher dose of chemotherapy causes many side 
affects, it is necessary that the total amount of chemotherapy agent should be minimized for minimization of the tumor size. At this point it is pertinent to be mentioned that we are interested to control only the chemotherapeutic drug as chemotherapy has more side effects than other two therapies. For that, we assume the chemotherapeutic drug is given as the function $u(t)$, which is time dependent. For the control variable $u(t)$, we consider a quadratic control to reflect the extremity of the imposed chemotherapeutic drugs' side effects. Therefore, the objective functional which is to be minimized is defined as:

$$
\Pi(u(t))=\int_{0}^{t_{f}}\left[\xi_{1} M+\frac{1}{2} \xi_{2} u^{2}\right] d t
$$

where the constants $\xi_{1}, \xi_{2}$ represent the weight factors of the respective terms which are used for balancing the size of the terms. The first term of the integrand signifies the number of cancerous cells and the second reflects the effectiveness of the drug. Here, we establish an optimal control $u^{*}$ such that

$$
\Pi\left(u^{*}\right)=\min \{\Pi(u): u \in \Omega\},
$$

where $\Omega=\left\{u: u\right.$ is measurable, $\left.0 \leq u(t) \leq 1, t \in\left[0, t_{f}\right]\right\}$ is the admissible control set. Hence, the problem with control $u(t)$ becomes

$$
\begin{aligned}
\frac{d I}{d t} & =q M+\frac{p I}{I+f}-\mu I+s-d_{1} I C \\
\frac{d M}{d t} & =r M(1-b M)-\frac{a I M}{g+M}-d_{2} M C \\
\frac{d C}{d t} & =u(t)-\gamma C
\end{aligned}
$$

with initial conditions,

$$
I(0)=I_{0}, \quad M(0)=M_{0}, \quad C(0)=C_{0}
$$

and objective functional

$$
\Pi(u(t))=\int_{0}^{t_{f}}\left[\xi_{1} M+\frac{1}{2} \xi_{2} u^{2}\right] d t
$$

\subsection{The existence of optimal control}

In this subsection, we will discuss the existence of an optimal control of our system (5.3). The solutions of the system (5.3) are bounded in a finite time interval. For proving this, we assume that

$$
\begin{gathered}
\vec{g}(t, \vec{X}, u)=\vec{\delta}(t, \vec{W})+\left(\begin{array}{c}
q \\
0 \\
u
\end{array}\right) \\
\text { with } \vec{W}=\left(\begin{array}{c}
I \\
M \\
C
\end{array}\right) \\
\qquad|\vec{g}(t, \vec{W}, u)| \leq\left|\left(\begin{array}{ccc}
p & 0 & 0 \\
0 & r & 0 \\
0 & 0 & -\gamma
\end{array}\right)\left(\begin{array}{c}
I \\
M \\
C
\end{array}\right)\right|+\left|\left(\begin{array}{c}
q \\
0 \\
u
\end{array}\right)\right|
\end{gathered}
$$


As the above system is linear having bounded coefficients in finite time interval, hence, we conclude that the solutions of the system (5.3) are bounded. By using the theorem proposed by Lukes [47] we get the admissible control class and the corresponding state equations with initial conditions (5.4) are found to be non-empty. Moreover, by definition of the set $\Omega$, it is clear that the control set $\Omega$ is convex and closed. Since the state solutions are bounded, hence, the continuity of R.H.S of the state system (5.3) holds and is bounded above by a sum of the bounded control and state.

Now, to prove the convexity of the integrand of $\Pi(u)$ on $\Omega$ we need to show

$$
\Pi[(1-\phi) u+\phi v] \leq[(1-\phi) \Pi(u)+\phi \Pi(u)]
$$

where $u, v$ are two distinct elements of $\Omega$ and $0 \leq \phi \leq 1$.

Now,

$$
\begin{aligned}
\Pi[(1-\phi) u+\phi v] & -[(1-\phi) \Pi(u)+\phi \Pi(u)] \\
= & \xi_{1} T(t)+\frac{\xi_{2}}{2}\{(1-\phi) u+\phi v\}^{2}-\left[(1-\phi)\left\{\xi_{1} T(t)+\frac{\xi_{2}}{2} u^{2}\right\}+\phi\left\{\xi_{1} T(t)+\frac{\xi_{2}}{2} v^{2}\right\}\right] \\
= & \frac{\xi_{2}}{2}\left(\phi^{2}-\phi\right)(u-v)^{2} \\
\leq & \quad\left[\text { since, } \phi \in[0,1] \text { and }(u-v)^{2}>0\right]
\end{aligned}
$$

which implies that the integrand of $\Pi(u)$ is convex on $\Omega$.

Again,

$$
\xi_{1} T(t)+\frac{1}{2} \xi_{2} u^{2}(t) \geq \frac{\xi_{2}}{2} u^{2}(t) \geq \frac{\xi_{2}}{2} u^{2}(t)-\eta_{2} \geq \eta_{1} u^{2}(t)-\eta_{2}
$$

Therefore, the integrand $\Pi(u)$ is bounded below by $\eta_{1} u^{2}(t)-\eta_{2}$. Hence, there exists an optimal control $u^{*}$ for which $\Pi(u)$ is minimized. From the above analysis, we established th following theorem.

Theorem 5.1. There exists an optimal control $u^{*}$ for a given objective functional

$$
\Pi(u(t))=\int_{0}^{t_{f}}\left[\xi_{1} M+\frac{1}{2} \xi_{2} u^{2}\right] d t
$$

such that $\Pi\left(u^{*}\right)=\min \{\Pi(u): u \in \Omega\}$, where $\Omega=\{u: u$ is measurable, $0 \leq u(t) \leq$ $\left.1, t \in\left[0, t_{f}\right]\right\}$ subject to the system (5.3) with initial conditions (5.4).

\subsection{Characterization of optimal control}

Pontryagins Maximum Principle [48] is invoked on the system (5.3) to derive the conditions for optimal control.

For the system (2.1) the Hamiltonian is defined as follows:

$$
\mathfrak{H}=\xi_{1} M+\frac{1}{2} \xi_{2} u^{2}+\theta_{1}\left[q M-\mu I-\frac{p I}{I+f}+s-d_{1} I C\right]+\theta_{2}\left[r M(1-b M)-\frac{a I M}{M+g}-d_{2} M C\right]+\theta_{3}[u-\gamma C],
$$

where, $\theta_{1}, \theta_{2}$ and $\theta_{3}$ are the adjoint functions which are to be determined. 
By using Pontryagins Maximum Principle [48] the adjoint system and the transversality conditions can be obtained as follows:

$$
\begin{aligned}
\frac{d \theta_{1}}{d t} & =-\left(\frac{\partial \mathfrak{H}}{\partial I}\right) \\
& =-\theta_{1}\left[-\mu+\frac{p f}{(I+f)^{2}}-d_{1} C\right]-\theta_{2}\left(\frac{-a M}{M+g}\right) \\
& =\theta_{1}\left[\mu-\frac{p f}{(I+f)^{2}}+d_{1} C\right]+\theta_{2} \frac{a M}{M+g} \\
\frac{d \theta_{2}}{d t} & =-\left(\frac{\partial \mathfrak{H}}{\partial M}\right) \\
& =-\xi_{1}-\theta_{1} q-\theta_{2}\left[r-2 r b M-\frac{a g I}{(M+g)^{2}}-d_{2} C\right] \\
& =\theta_{2}\left[2 r b M+\frac{a g I}{(M+g)^{2}}+d_{2} C-r\right]-\theta_{1} q-\xi_{1} \\
\frac{d \theta_{3}}{d t} & =-\left(\frac{\partial \mathfrak{H}}{\partial C}\right) \\
& =\theta_{1} d_{1} I+\theta_{2} d_{2} M+\theta_{3} \gamma
\end{aligned}
$$

The transversality conditions are

$$
\theta_{1}\left(t_{f}\right)=0, \quad \theta_{2}\left(t_{f}\right)=0, \quad \theta_{3}\left(t_{f}\right)=0 .
$$

From the optimality condition of $u$, we have

$$
\begin{aligned}
\frac{\partial \mathfrak{H}}{\partial u} & =\xi_{2} u^{*}+\theta_{3}=0 \quad\left(\text { at } u_{1}=u_{1}^{*}(t)\right) \\
\Longrightarrow u^{*}(t) & =-\frac{\theta_{3}}{\xi_{2}}
\end{aligned}
$$

By using the bounds of control $u_{1}(t)$, we get

$$
u^{*}= \begin{cases}-\frac{\theta_{3}}{\xi_{2}}, & \text { if } 0 \leq-\frac{\theta_{3}}{\xi_{2}} \leq 1 \\ 0, & \text { if }-\frac{\theta_{3}}{\xi_{2}} \leq 0 \\ 1, & \text { if }-\frac{\theta_{3}}{\xi_{2}} \geq 1\end{cases}
$$

Hence, from the above discussion, we get the following result:

Theorem 5.2. Given an optimal control $u^{*}$ with the corresponding state variables $I^{*}(t)$, $M^{*}(t), C^{*}(t)$, there exist co-states or adjoint variables $\theta_{1}, \theta_{2}$, and $\theta_{3}$ satisfying the following system:

$$
\begin{aligned}
\frac{d \theta_{1}}{d t} & =\theta_{1}\left[\mu-\frac{p f}{(I+f)^{2}}+d_{1} C\right]+\theta_{2} \frac{a M}{M+g} \\
\frac{d \theta_{2}}{d t} & =\theta_{2}\left[2 r b M+\frac{a g I}{(M+g)^{2}}+d_{2} C-r\right]-\theta_{1} q-\xi_{1} \\
\frac{d \theta_{3}}{d t} & =\theta_{1} d_{1} I+\theta_{2} d_{2} M+\theta_{3} \gamma
\end{aligned}
$$


with transversality conditions

$$
\theta_{1}\left(t_{f}\right)=0, \quad \theta_{2}\left(t_{f}\right)=0, \quad \theta_{3}\left(t_{f}\right)=0 .
$$

Moreover,

$$
u^{*}=\min \left\{\max \left\{0,-\frac{\theta_{3}}{\xi_{2}}\right\}, 1\right\}
$$

In the next section, we have solved our system (2.1) and (5.3) both numerically and graphically.

\section{$6 \quad$ Numerical Simulation}

The following simulations have been done using the parameters of Table 1 . We take the external immunotherapy $s(t)$ as the key parameter for analysis of the model. The time series diagrams of effector cells, tumor cells, and drug administration without control for different values of $s(t)$ but with the same cancer clearance term have been presented in Figure 2, Figure 3, and Figure 4. In Figure 2, it is seen that while the cancer clearance term is $a(t)=5$ during gene therapy with low immunity $s(t)=1$, both effector and tumor cells go in tandem in the presence of chemotherapy. In this situation, there are two biologically feasible solutions $E_{1}(37.48,0,1.65)$ and $E_{5}(965.34,556.72,1.65)\left(u_{0}=\right.$ 0.01650 ) having unstable behavior. In our second observation in Figure 3 with $a(t)=5$ and moderate level of immunotherapy $s(t)=25$, the system has two feasible equilibrium points $E_{2}(837.483,0,1.65)$ and $E_{6}(960.709,73.935,1.65)\left(u_{0}=0.01650\right)$ which are again found to be unstable. In Figure 4 we have observed that with high immunity $s(t)=53$, the cancer clearance term of gene therapy $a(t)=5$, and in the presence of chemo-drug, the tumor cell goes to zero rather than oscillating about zero in time around $t=120$ and the effector cells converge to a stable position. This scenario implies that there are only one biologically valid equilibrium $E_{2}(1770.82,0,1.65)\left(u_{0}=0.01650\right)$, which is locally stable in nature.

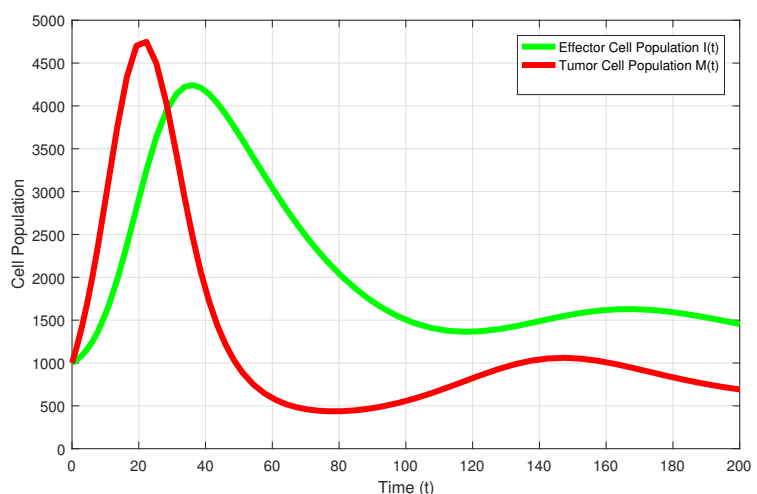

(a) Time series plot for cell population

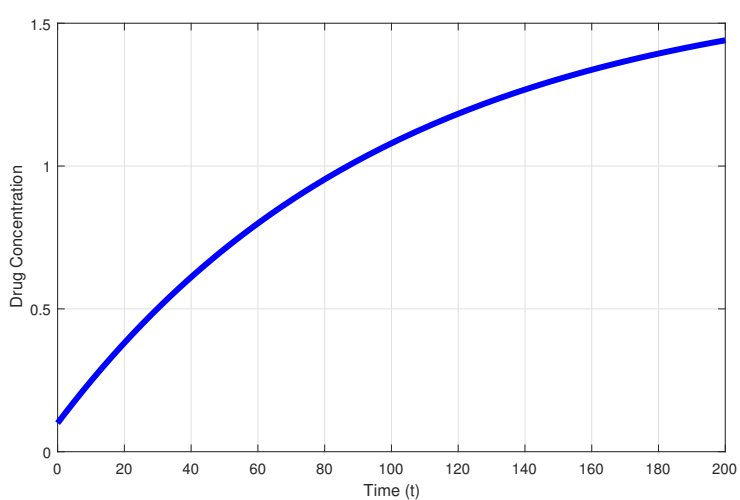

(b) Chemotherapy drug administration

Figure 2: Combination treatment with immunotherapy term $s(t)=1$ (without chemotherapy drug control) 


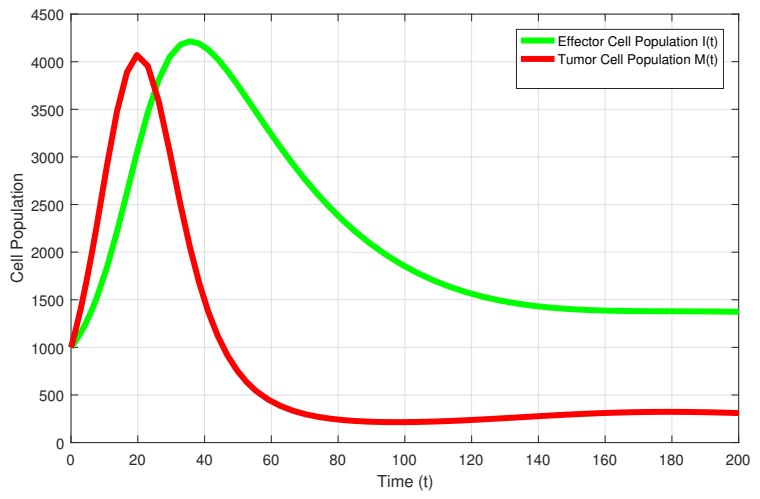

(a) Time series plot for cell population

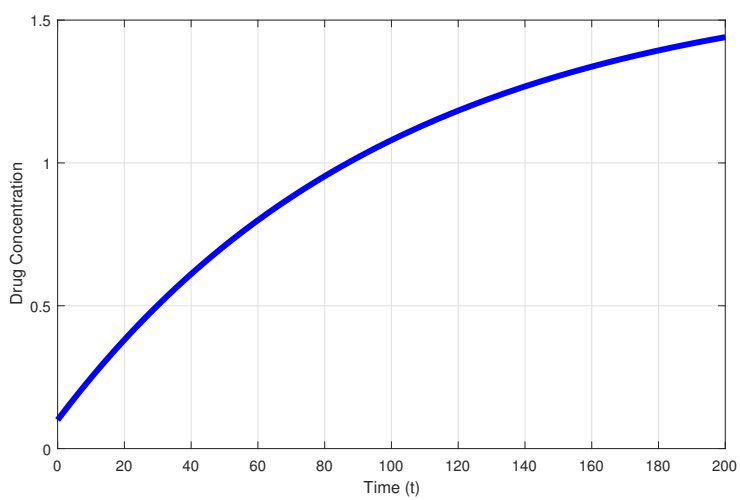

(b) Chemotherapy drug administration

Figure 3: Combination treatment with immunotherapy term $s(t)=25$ (without chemotherapy drug control)

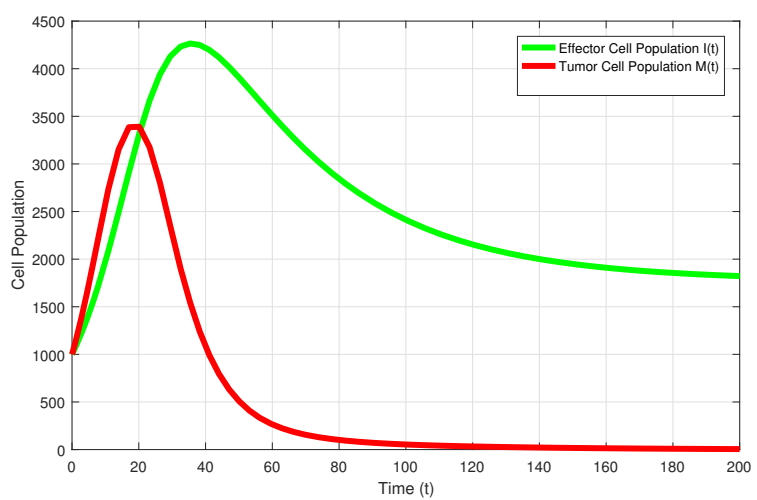

(a) Time series plot for cell population

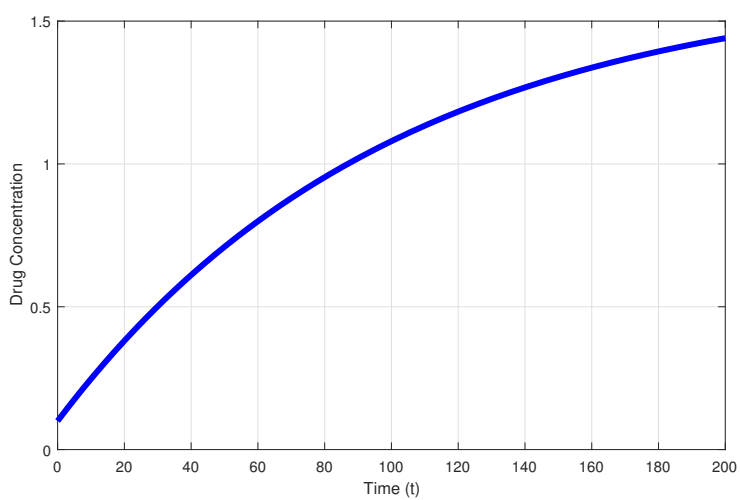

(b) Chemotherapy drug administration

Figure 4: Combination treatment with immunotherapy term $s(t)=53$ (without chemotherapy drug control) 
Table 1: Value of the parameters using in the simulation

\begin{tabular}{|c|c|c|c|c|}
\hline Parameters & Meaning & Values & interval & Source \\
\hline$q(t)$ & cancer antigenicity & $0.05(1 /$ time $)$ & {$\left[10^{-3}, 0.5\right]$} & {$[33]$} \\
\hline$s(t)$ & Immunotherapy term & {$\left[10^{-2}, 10^{2}\right]$} & {$\left[10^{-2}, 10^{2}\right]$} & {$[33]$} \\
\hline$p$ & proliferation rate of $I$ & $0.1245(1 /$ time $)$ & 0.1245 & {$[33]$} \\
\hline$f$ & $\begin{array}{l}\text { Half saturation for } I \\
\text { proliferation term }\end{array}$ & $10^{-3}($ cells $)$ & {$\left[10^{-5}, 1\right]$} & {$[33]$} \\
\hline$\mu$ & half life of effector cells $I$ & $0.03(1 /$ time $)$ & 0.03 & {$[33]$} \\
\hline$r(t)$ & cancer growth rate & $0.18(1 /$ time $)$ & {$\left[10^{-1}, 2\right]$} & {$[33]$} \\
\hline$b$ & cancer cell capacity & $10^{-9}(1 /$ cells $)$ & $10^{-9}$ & {$[33]$} \\
\hline$a(t)$ & cancer clearance term & 5 & {$\left[10^{-2}, 10^{2}\right]$} & {$[33]$} \\
\hline$g$ & $\begin{array}{l}\text { Half saturation for } \\
\text { cancer clearance }\end{array}$ & $10^{5}($ cells $)$ & $10^{5}$ & {$[33]$} \\
\hline$d_{1}$ & $\begin{array}{l}\text { Response Coefficients of } \\
\text { Chemotherapy drugs to } I\end{array}$ & $2 \times 10^{-11} /$ day & $2 \times 10^{-11} /$ day & {$[27]$} \\
\hline$d_{2}$ & $\begin{array}{l}\text { Response Coefficients of } \\
\text { Chemotherapy drugs to } M\end{array}$ & $0.08 /$ day & $0.08 /$ day & {$[27]$} \\
\hline$\gamma$ & $\begin{array}{l}\text { Decay rate of } \\
\text { Chemotherapy drug }\end{array}$ & $0.01 /$ day & $0.01 /$ day & {$[27]$} \\
\hline
\end{tabular}

The optimal system (5.3) associated with the adjoint system (5.16) and the separated boundary conditions at time $t=0$ and $t=t_{f}$ are solved. Forward method is used to solve the original optimal system (5.3) and the backward method for the respective adjoint system (5.16) with $t_{f}=200$. Here, we consider the treatment parameters $a(t)=5$, $s(t)=53$ and the control of drug $u(t)$. As the variables associated with the optimal system (5.3) and in the objective functional (5.5) have different scales, hence, to balance the objective functional, we have chosen the weight constants $\xi_{1}=5, \xi_{2}=10$ in (5.5). In Figures 5(a) and 5(b), we have presented the time series plots of effector cells and tumor cells with control. We have seen that the tumor cells vanish around time $15<t_{f}<20$. Figure 6 shows that the drug concentration reduces gradually depending upon the size of the tumor and hence it also goes to zero as the tumor vanishes from the body.

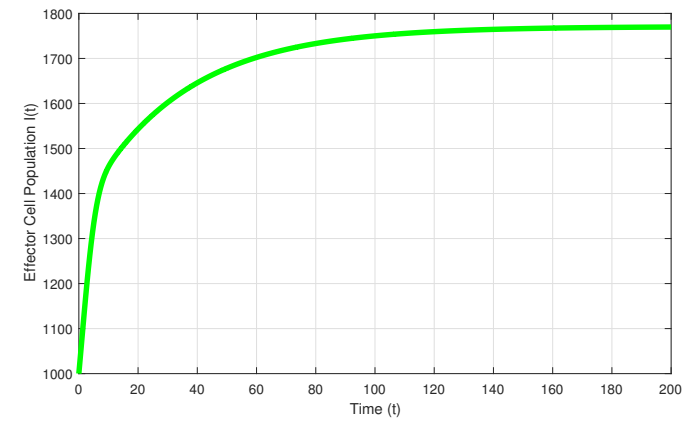

(a) Time series plot for effector cell population with chemotherapy drug control $u$

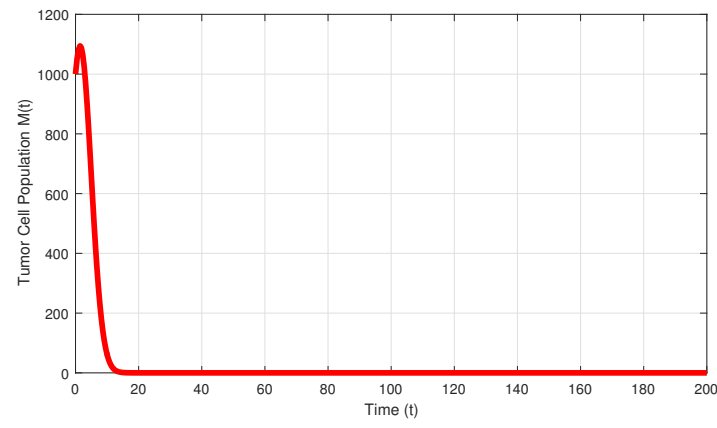

(b) Time series plot for tumor cell population with chemotherapy drug control $u$ 


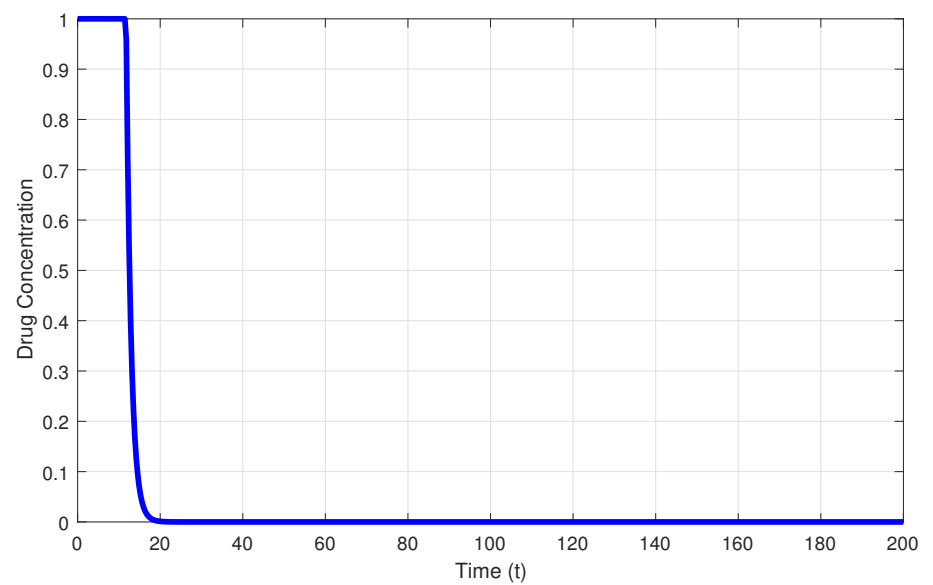

Figure 6: Graph for the Optimal chemotherapeutic drug control $u$

\section{Conclusion}

We have developed an ODE mathematical model for tumor growth under the action of effector immune cells in the presence of gene therapy, immunotherapy and chemotherapy. We established the basic characteristics of the dependent variables of the model. We presented the stability analysis of the system, revealing the conditions that must hold well for the cure equilibrium to be attained. For the numerical solution, we set up $s$ as the key parameter. We investigated the dynamics of the model for the values (i) $s(t)=1$, (ii) $s(t)=25$, and (iii) $s(t)=53$, respectively. From the numerical simulation, it can be concluded that for the value of immunotherapy term $s(t)=53$, in the presence of gene and chemotherapy, tumor cells may eradicate from the body. In [33], the authors found the values of immunotherapy term as $s(t)=100 ; s(t)=764.5072$ and cancer clearance term as $a(t)=2 ; a(t)=38.0040$ depending upon whether the sufficient conditions of global stability of tumor free steady state is satisfied or not. However, our results reveal that in the presence of chemotherapy the patient requires less amount of external source of immunotherapy to fight against cancer. This conclusion can be drawn by comparing our results with those of [33].

We also have constructed an optimal control problem associated with the system to minimize the tumor cell population and the chemotherapeutic drug within a finite time interval $[0,200]$. According to our results, the tumor cells can be eliminated from the body in a short period of time in the presence of chemo-immuno-gene therapy with the control of chemotherapy drug parameter $u(t)$. Our model is probably one of the very few models in which gene therapy is combined with chemo-immuno therapy for cancer treatment. Our future work is to see a realistic scenarios by using fractional derivative to model and investigate the corresponding control problem.

\section{Declaration}

- Availability of data and materials

Not applicable. 


\section{- Competing interests}

The authors declare that they have no competing interests.

\section{- Funding}

There is no funding for this work.

\section{- Authors' contribution}

All authors jointly worked on the results and they read and approved the final manuscript.

\section{- Acknowledgements}

Not applicable.

\section{References}

[1] Bellomo N., Li N.K., Maini P.K., On The Foundations of Cancer Modelling: Selected Topics, Speculations, and Perspectives, Mathematical Models and Methods in Applied Sciences, Vol. 18, No. 4, 2008, 593646.

[2] Araujo R.P., McElwain D.L.S., A history of the study of solid tumour growth: the contribution of mathematical modelling, Bull. Math. Biol. 66, 10391091 ,2004.

[3] Kuznetsov V.A., Makalkin I.A., Taylor M.A., Perelson A.S., Nonlinear dynamics of immunogenic tumors: parameter estimation and global bifurcation analysis, Bulletin of Mathematical Biology, Vol. 56, No. 2, pp. 295-321, 1994.

[4] Kirschner D., Panetta J.C., Modeling immunotherapy of the tumor-immune interaction, J. Math. Bio., 37, 1998, 235252.

[5] Kolev M., Kozlowska E., Lachowicz M., A Mathematical Model for Single Cell CancerImmune System Dynamics, Mathematical and Computer Modelling, 41, 2005 10831095 .

[6] Berezhnaya N.M., Interaction between tumor and immune system: the role of tumor cell biology, Exp Oncol., 2010;32(3):159-166.

[7] Gonzalez H., Hagerling, C., Werb Z., Roles of the immune system in cancer: From tumor initiation to metastatic progression, Genes Dev., 2018, 32, 12671284.

[8] Rihan F.A., Safan M., Abdeen M.A., Rahman D.A., Qualitative and Computational Analysis of a Mathematical Model for Tumor-Immune Interactions, Journal of Applied Mathematics, Volume 2012, Article ID 475720, 19 pages, doi:10.1155/2012/475720.

[9] Bi P., Ruan S., Zhang X., Periodic and chaotic oscillations in a tumor and immune system interaction model with three delays, Chaos: An Interdisciplinary Journal of Nonlinear Science, 24, 023101 (2014); doi: 10.1063/1.4870363

[10] Khajanchi S., Banerjee S., Stability and bifurcation analysis of delay induced tumor immune interaction model, Applied Mathematics and Computation, 248, (2014), 652671. http://dx.doi.org/10.1016/j.amc.2014.10.009 
[11] Khajanchi S., Perc M., Ghosh D., The influence of time delay in a chaotic cancer model, Chaos, 28, 103101 (2018); doi: 10.1063/1.5052496

[12] Khajanchi S., Chaotic dynamics of a delayed tumor-immune interaction model, International Journal of Biomathematics, (2019) doi: 10.1142/S1793524520500096

[13] Ghosh D., Khajanchi S., Mangiarotti S., Denis F., Dana S.K., Letellier C., How tumor growth can be influenced by delayed interactions between cancer cells and the microenvironment?.BioSystems, Elsevier, 2017, 158, pp.17 - 30. 10.1016/j.biosystems.2017.05.001, hal-01672073.

[14] Ruan S., Nonlinear Dynamics In Tumor-Immune System Interaction Models With Delays, DISCRETE AND CONTINUOUS DYNAMICAL SYSTEMS SERIES B, Volume 26, Number 1, pp. 541- 602, January 2021 doi:10.3934/dcdsb.2020282

[15] Vincent T., DeVita Jr., Edward C., A History of Cancer Chemotherapy, Cancer Res, November 12008 (68) (21) 8643-8653; DOI: 10.1158/0008-5472.CAN-07-6611.

[16] De Pillis L.G., Fister K.R., Gu W., Head T., Maples K., Neal T., Murugan A., Kozai K., Optimal Control of Mixed Immunotherapy and Chemotherapy of Tumors, Journal of Biological Systems, Vol. 16, No. 1, 2008, 5180.

[17] de Pillis et al., Mathematical model creation for cancer chemo-immunotherapy., Computational and Mathematical Methods in Medicine, Vol. 10, 2009, No. 3, 165 184.

[18] Pinho S.T.R., Bacelar F.S., Andradea R.F.S., Freedman H.I., A mathematical model for the effect of anti-angiogenic therapy in the treatment of cancer tumours by chemotherapy., Nonlinear Analysis: Real World Applications., 14, 2013, 815 - 828.

[19] Ghaffari A., Nazari M., Arab F., Optimal finite cancer treatment duration by using mixed vaccine therapy and chemotherapy: state dependent Riccati equation control, Journal of Applied Mathematics, 2014; 3:19.

[20] Liu Z., Yang C., A mathematical model of cancer treatment by radiotherapy followed by chemotherapy, Mathematics and Computers in Simulation, 124 (2016), 115.

[21] Guiraldello R.T., Martins M.L., Manceraa P.F.A., Evaluating the efficacies of Maximum Tolerated Dose and metronomic chemotherapies: A mathematical approach, Physica A, 456, 2016, 145-156.

[22] Pang L., Shen L., Zhao Z., Mathematical Modelling and Analysis of the Tumor Treatment Regimens with Pulsed Immunotherapy and Chemotherapy, Computational and Mathematical Methods in Medicine, Article ID 6260474, 12 pages, 2016.

[23] Rodrigues D.S., Mancera P.F.A., Carvalho T., Gonalves L.F., A mathematical model for chemoimmunotherapy of chronic lymphocytic leukemia, Applied Mathematics and Computation, 349, 2019, 118133.

[24] Gil W.F.F.M., Carvalho T., Mancera P.F.A., Rodrigues D.S., A Mathematical Model on the Immune System Role in Achieving Better Outcomes of Cancer Chemotherapy, Tendencias em Matematica Aplicada e Computacional, 20, N. 2 (2019), 343-357. doi: 10.5540/tema.2019.020.02.0343 
[25] Alqudah M.A., Cancer treatment by stem cells and chemotherapy as a mathematical model with numerical simulations, Alexandria Engineering Journal, (2020), https://doi.org/10.1016/j.aej.2019.12.025

[26] Khajanchi S., Ghosh D., The combined effects of optimal control in cancer remission, Applied Mathematics and Computation, Volume 271, 15 November 2015, Pages 375388, https://doi.org/10.1016/j.amc.2015.09.012

[27] Sharma S., Samanta G.P., Dynamical Behaviour of a Tumor-Immune System with Chemotherapy and Optimal Control, Journal of Nonlinear Dynamics, Volume 2013, Article ID 608598, 13 pages, http://dx.doi.org/10.1155/2013/608598.

[28] Maurici D., Hainaut P., TP53 gene and p53 protein as targets in cancer management and therapy,BIOTECHNOLOGY, 2001;12.

[29] Yardley D.A., Drug Resistance and the Role of Combination Chemotherapy in Improving Patient Outcomes, International Journal of Breast Cancer, vol. 2013, Article ID 137414, 15 pages, 2013. https://doi.org/10.1155/2013/137414

[30] Zhang M., Garbuzenko B.O., Reuhl R.K., Rodriguez-Rodriguez L., Minko T., Twoin-one: combined targeted chemo and gene therapy for tumor suppression and prevention of metastases, Nanomedicine, (2012) 7(2), 185197.

[31] Lin T., Zhang L., Davis J., Gu J., Nishizaki M., Ji L., Roth A.J., Xiong M., Fang B., Combination of TRAIL Gene Therapy and Chemotherapy Enhances Antitumor and Antimetastasis Effects in Chemosensitive and Chemoresistant Breast Cancers, MOLECULAR THERAPY, Vol. 8, No. 3, September 2003.

[32] Cannon L., Hernandez M.J., Zurakowski R., Modeling and analysis of genetherapeutic combination chemotherapy for pancreatic cancer, Proceedings of the 18th World Congress, The International Federation of Automatic Control, Milano (Italy), August 28 - September 2, 2011.

[33] Tsygvintsev A., Marino S., Kirschner D. E., A Mathematical Model of Gene Therapy for The Treatment of Cancer, Springer-Verlag, Berlin-Heidelberg-New York, 2013.

[34] Rihan F.A., Abdelrahman D.H., Al-Maskari F., Ibrahim F., Abdeen M.A., Delay differential model for tumour-immune response with chemoimmunotherapy and optimal control, Computational and Mathematical Methods in Medicine, vol. 2014, Article ID 982978, 15 pages, 2014.

[35] d'Onofrio A., Ledzewicz U., Maurer H., Schttler H., On optimal delivery of combination therapy for tumors, Mathematical Biosciences, 222, 2009, 1326.

[36] Khajanchi S., Stability Analysis of a Mathematical Model for Glioma-Immune Interaction under Optimal Therapy, Int. J. Nonlinear Sci. Numer. Simul., 20(34), (2019), 269285.

[37] Leszczynski M., Ledzewicz U., Schattler H., Optimal Control For A Mathematical Model For Chemotherapy With Pharmacometrics, Math. Model. Nat. Phenom., 15, 2020, 69. https://doi.org/10.1051/mmnp/2020008 
[38] Khalili P., Vatankhah R., Derivation of an optimal trajectory and nonlinear adaptive controller design for drug delivery in cancerous tumor chemotherapy, Computers in Biology and Medicine, 2019, https://doi.org/10.1016/j.compbiomed.2019.04.011.

[39] Najafi M., Basirzadeh H., Optimal control homotopy perturbation method for cancer model, International Journal of Biomathematics, Vol. 12, No. 2, 2019, 1950027 (12 pages), DOI: 10.1142/S179352451950027X.

[40] Rihan F.A., Lakshmanan S., Maurer H., Optimal control of tumour-immune model with time-delay and immuno-chemotherapy, Applied Mathematics and Computation, 353, (2019), 147165, https://doi.org/10.1016/j.amc.2019.02.002.

[41] Bukkuri A., Optimal control analysis of combined chemotherapy-immunotherapy treatment regimens in a PKPD cancer evolution model, Biomath, 9, 2020, 2002137, http://dx.doi.org/10.11145/j.biomath.2020.02.137

[42] Das P., Das S., Upadhyay R.K., Das P., Optimal treatment strategies for delayed cancer-immune system with multiple therapeutic approach, Chaos, Solitons and Fractals, 136, (2020), 109806. https://doi.org/10.1016/j.chaos.2020.10980

[43] Lestari D., Dwi Ambarwati R., A Local Stability of Mathematical Models for Cancer Treatment by Using Gene Therapy, International Journal of Modeling and Optimization, Vol. 5, No. 3, June 2015.

[44] Margarit D. H., Romanelli L., (2015). A Simple Model For Control Of Tumor Cells. Journal of Biological Systems, 23(supp01), S33S41. doi:10.1142/s0218339015400033

[45] Wilson S., Levy D., A mathematical model of the enhancement of tumour vaccine ecacy by immune therapy. BMB, 2012 Mar; 74(7):1485500. PMid: 22438084, PMCid: PMC3822329. https://doi.org/10.1007/s11538-012-9722-4

[46] Frascoli F., Kim P.S., Hughes B.D., Landman K.A., A dynamical model of tumour immunotherapy, Mathematical Biosciences, 2014; 253:5062. PMid: 24759513. https://doi.org/10.1016/j. mbs.2014.04.003

[47] Lukes D.L., Differential Equations : Classical to Controlled, Mathematics in Science and Engineering, Academic Press, NewYork,1982.

[48] Pontryagin L.S., Boltyanskii V.G., Gamkrelidze R.V., Mishchenko E.F., The Mathematical Theory of Optimal Process, Gordon and Breach, 1962. 